\title{
Report of 4 cases of vulvar lymphangioma: an update
}

\begin{abstract}
Vulvar Lymphangioma is an infrequent pathology. There are few cases published in the English literature. We present 4 new cases of vulvar lymphangioma (1 acquired and 3 congenital) treated at the vulvar section of Hospital Italiano de Buenos Aires. It represents a challenge for clinicians because of its rarity. The main differential diagnosis is genital warts. This disease has cosmetic and psychosexual implications in the patients; therefore surgery with a cosmetic approach is the recommended treatment. Labiaplasty of labia majora and laser $\mathrm{CO} 2$ vaporization have similar success and recurrence rates. Conservative management (observation) is a valid option if the patient is asymptomatic.
\end{abstract}

Keywords: acquired lymphangioma, benign vulvar tumors, congenital lymphangioma, vulvar lymphangioma, vulvar lymphangioma, management
Volume 9 Issue 2 - 2018

\author{
Maria C Sluga,' Maria M Domenech,' Maria \\ S Peremateu,' Maria L Absi,' Alejandra \\ Wernicke, ${ }^{2}$ Claudia E Marchitelli ${ }^{3}$ \\ 'Department of Gynecology, Hospital Italiano de Buenos Aires, \\ Argentina \\ ${ }^{2}$ Department of Pathology, Hospital Italiano de Buenos Aires, \\ Argentina \\ ${ }^{3}$ Chief of Vulvar Section, Department of Gynecology, Hospital \\ Italiano de Buenos Aires, Argentina
}

Correspondence: Claudia E Marchitelli, Chief of Vulvar Section. Department of Gynecology, Hospital Italiano de Buenos Aires, Av Pavan 4239 CABA, Zip Code 1428, Buenos Aires, Argentina, Tel 54 || 49225267.

Email Claudia.marchitelli@ospitalitaliano.org.ar

\section{Introduction}

Lymphangioma is a rare, non-epithelial, benign disease of the vulva. The most frequent type of lymphangioma that can present vulvar localization is Lymphangioma Circumscriptum (LC). Lymphangioma Circumscriptum can be classified according to its origin as primary (in approximately one third of cases) or more frequently as secondary (or acquired). Below, we present three cases of congenital vulvar LC and one case of acquired vulvar LC, the latter being associated to chronic lymphedema of the lower extremities.

\section{Case reports}

\section{Case 1: Acquired vulvar lymphangioma circumscriptum}

This case describes a 45 -year-old patient who was referred to the department of Vulvar Disease with a previous diagnosis of giant vulvar condylomatosis. The patient's main complaints included vulvar pain, difficulty performing her daily chores and discomfort during intercourse. Upon physical examination, severe elephantiasis of both legs was evident. Vulvar inspection revealed a large tumorous formation which consisted of multiple small confluent vesicles that replaced the normal vulvar labial anatomy from the clitoris to the fourchette, predominantly on the right side. Hypertrophy of the labia minora was also evident (Figure 1). Because of her general lymphedema a presumptive diagnosis of lymphangioma was made and the initial diagnosis of condyloma was discarded (Figure 2). Upon biopsy, clear serous discharge emerged from the vesicles. Histopathology reported abnormal changes related with acute eczema. Vulvar lymphangioma with elephantiasis secondary to chronic lymphedema was diagnosed based on clinical findings. Bilateral major labioplasty was performed for symptom relief and to improve cosmetic. The diagnosis was confirmed by histology. The pathologic report of the surgical specimen described a microcytic lymphatic malformation (lymphangioma) (Figure 3). Recurrence was seen after the first year. Laser $\mathrm{CO} 2$ vaporization was the chosen treatment with an excellent outcome. After eight years, a second recurrence was observed. Vesicles appeared across a small patch of skin on the left labia majora and the right perianal area, and a second surgery with a cosmetic approach was performed.

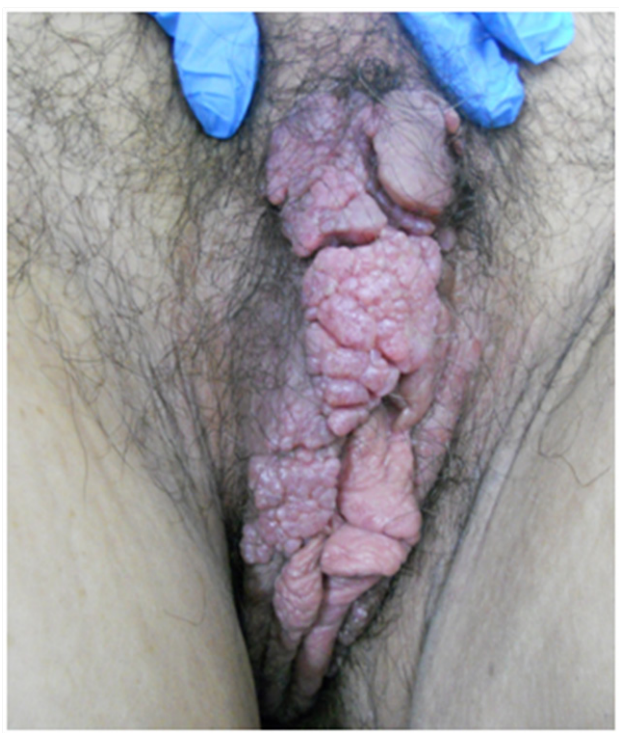

Figure I Case I: Acquired vulvar lymphangioma circumscriptum. Multiple confluent vesicles smaller than $4 \mathrm{~mm}$ replaced both labia minora.

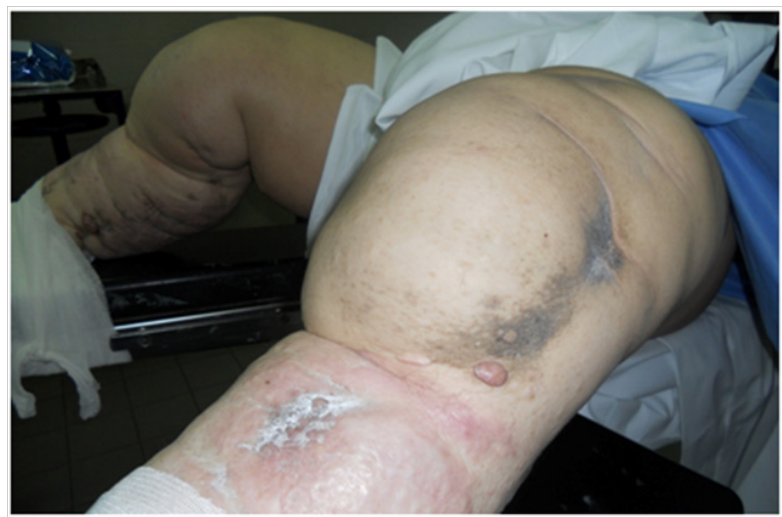

Figure 2 Case I: Bilateral lymphedema affecting both legs. 


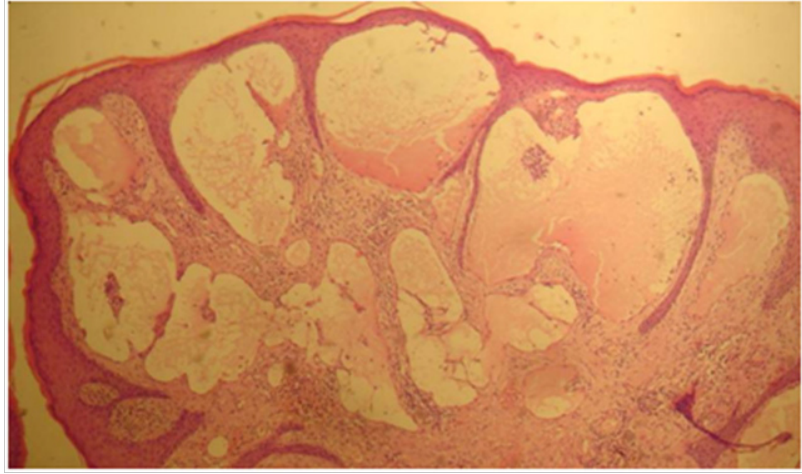

Figure 3 Case I: Histology: Dilated vessels coated by a monolayer of endothelial cells with eosinophil and amorphous content.

\section{Case 2: Congenital vulvar circumscribed lymphangioma}

This case describes a 24-year-old patient with a history of congenital lymphatic disease of the right leg. She had already undergone surgery for this disorder 2 years earlier. She was referred by her gynecologist to the vulvar disease department with a presumptive diagnosis of recurrent perianal condyloma. Her main complaint was pruritus and presence of palpable perianal lesions (Figure 4). She had previously received superficial laser $\mathrm{CO} 2$ vaporization. Upon physical examination, surgical scars were visible on her right leg and a series of confluent translucent vesicles were identified across the right perianal region. These lesions were ipsilateral to the previously affected lower limb. Because of her underlying pathology and the clinical aspect of the lesions, lymphangioma was suspected. Biopsy of the lesions was performed and histopathology confirmed the diagnosis of LC. The patient underwent Laser CO2 vaporization down to the deep dermis. At 48 months postoperative, she remains lesion-free.

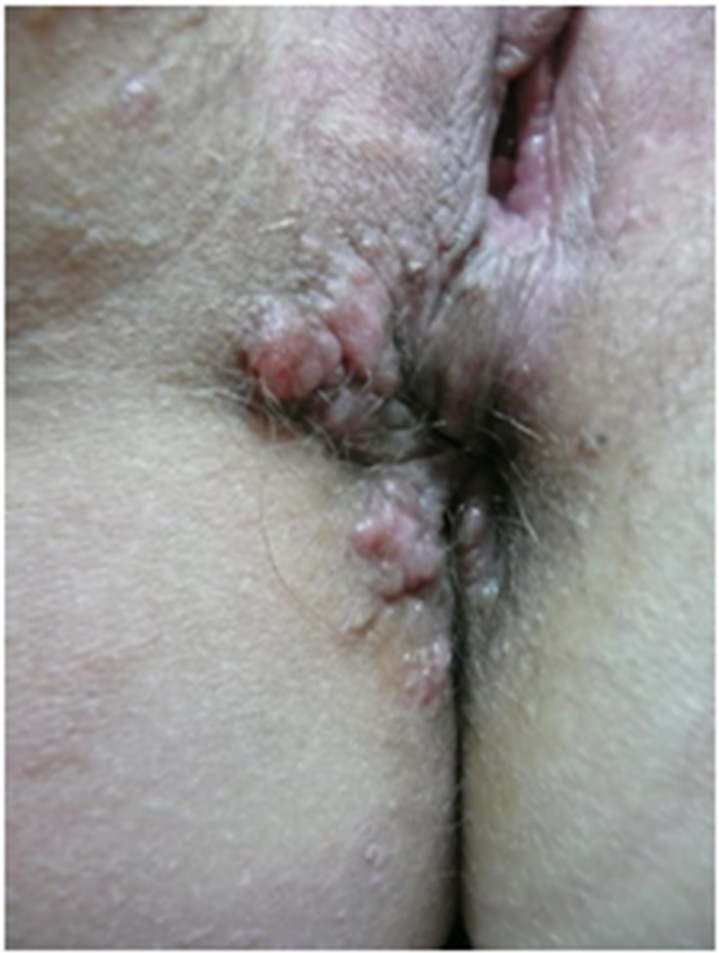

Figure 4 Case 2: Congenital vulvar circumscribed lymphangioma: confluent growths of bullous aspect, translucent, soft to the touch vesicles at the right side of the perianal area.

\section{Case 3: Congenital vulvar circumscribed lymphangioma}

This case describes a 68-year-old patient, with a long history of vulvar pruritus, who had previously received topical treatments for her symptoms with steroid and antibiotic lotions on multiple occasions with poor clinical response. A biopsy of the lesion was performed. Pathology report informed LC. The patient was referred to our department for treatment. Upon physical examination, both labia majora were covered with small papules (smaller than $2 \mathrm{~mm}$ ), with crystalline clear content (Figure 5). These clinical findings were consistent with the pathology report. A combined therapeutic approach of surgical excision followed by $\mathrm{CO} 2$ laser vaporization was performed. The patient is currently 84 months into follow up and has not shown signs of recurrence to date.

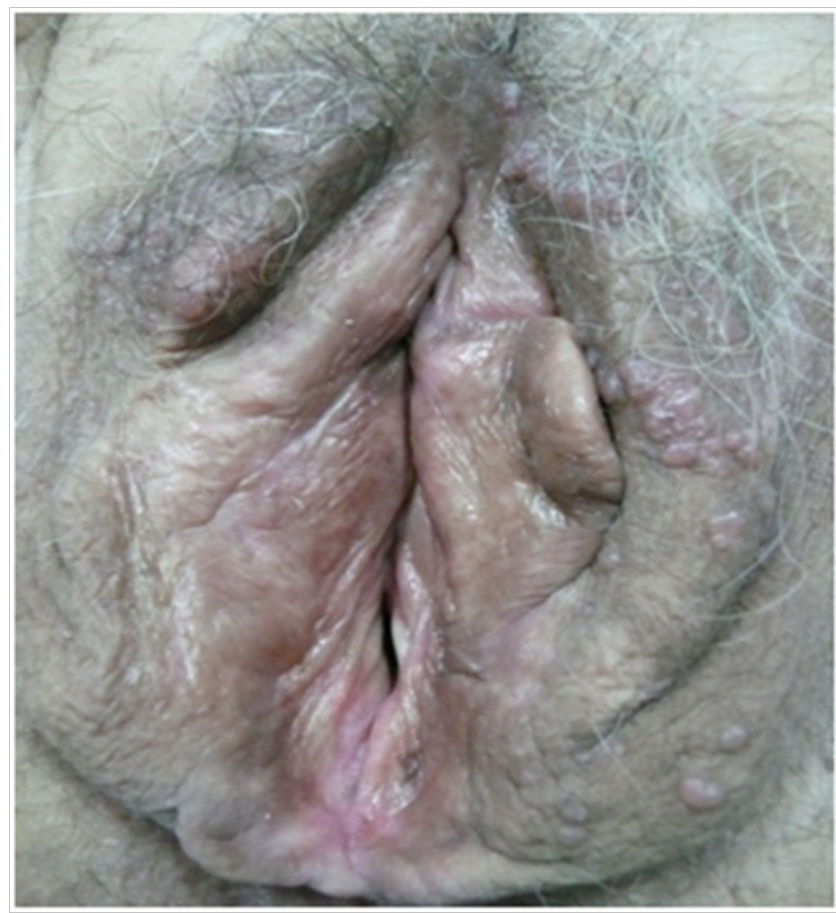

Figure 5 Case 3: Congenital vulvar circumscribed lymphangioma: small papules $(2 \mathrm{~mm})$, with crystalline clear content, distributed all over the labia majora.

\section{Case 4: Congenital vulvar circumscribed lymphangioma}

This is the case of a 64-year-old patient who was referred to the department of vulvar disease for evaluation of a tumor on the mons pubis. She reported having the lesion for over five years, but referred that over the past month she had begun to experience pain and pruritus accompanied by foul discharge. Upon physical examination, several confluent tumors were observed some with clinical signs of infection, edema and odorous yellow discharge (Figure 6). Oral antibiotic therapy with amoxicillin-clavulanic acid was prescribed for 7days and a biopsy of the lesion was performed. The patient referred improvement of symptoms and the infection was adequately controlled. Pathology report of the biopsy informed LC. After the infection resolved, $\mathrm{CO} 2$ laser vaporization was recommended. The patient decided not to undergo treatment and is currently in follow up.

\section{Discussion}

Lymphangioma is a rare, benign pseudo tumor. It is a malformation of the lymphatic system and not a true neoplasia. It represents 
approximately 1 to $4 \%$ of all vascular malformations and $26 \%$ of all benign vascular tumors. Although it can develop almost anywhere in the body except the central nervous system, $95 \%$ of lymphangioma occur in the skin and subcutaneous cellular tissue of the head, neck and axillary region. ${ }^{1}$ The remaining $5 \%$ can be distributed over the rest of the body, although vulvar localization is extremely rare. ${ }^{2}$

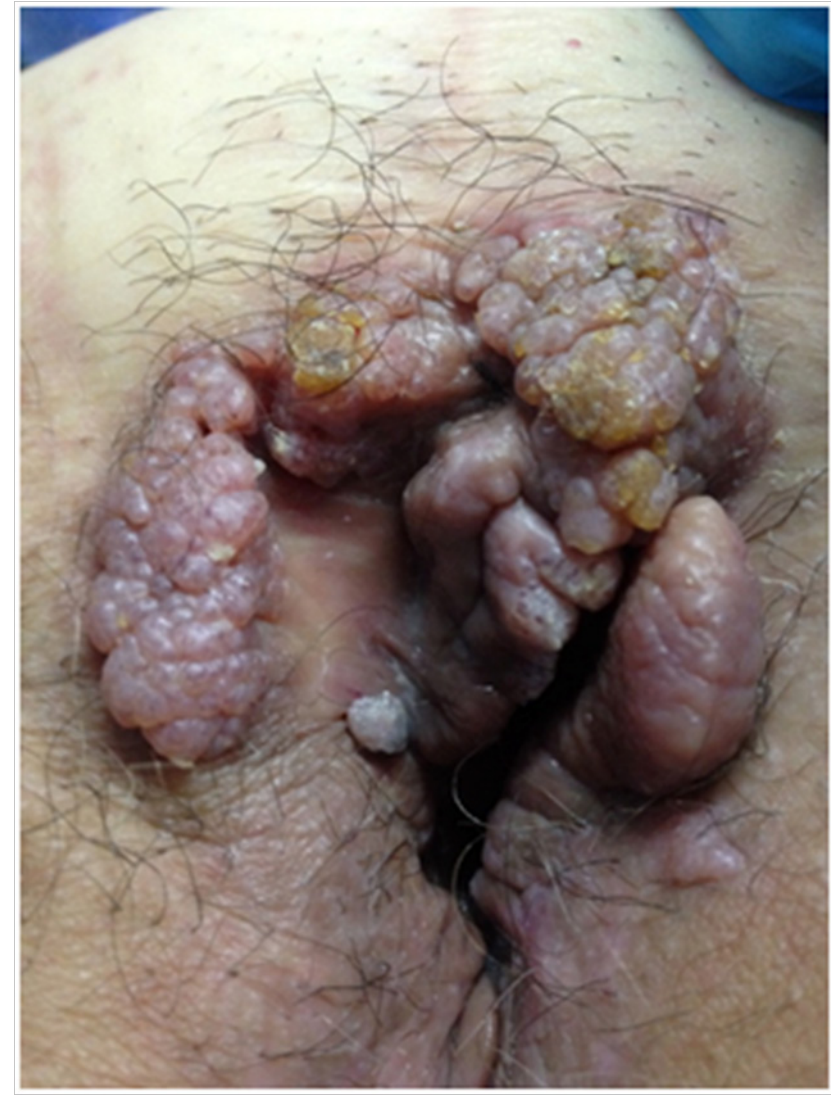

Figure 6 Case 4: Congenital vulvar circumscribed lymphangioma: confluent tumors, signs of wound infection, edema and a smelly yellowish discharge.

The term "circumscriptum" was first used by Malcolm Morris in $1889 .{ }^{3}$ The type of lymphangioma that most frequently develops in the vulva is circumscriptum. Lymphangioma Circumscriptum is located superficially within the skin and is composed of small thinwalled lymphatic vessels. It can be congenital (primary) or acquired (secondary). This last classification is the one most frequently used in scientific literature. It is generally accepted that lymphangiectasis is clinically and histologically indistinguishable from superficial lymphangioma and also tends to develop in the areas affected by obstructed or destroyed lymphatic drainage., ${ }^{4,5}$

The etiology of LC is still not clear. Congenital LC (CLC), is believed to be caused by developmental malformations of the lymphatic vessels in the papillary dermis and the subcutaneous tissue ${ }^{3}$, that are excluded from the general lymphatic system, causing stasis and build up. ${ }^{6}$ The clinically evident vesicles are the projection of these dilated lymphatic vessels into the papillary dermis. ${ }^{6}$ Regarding acquired LC (ALC), the cause is believed to be the newly onset obstruction of previously normally developed lymphatic vessels. These newly obstructed vessels cannot drain into deep lymphatic networks, interfering with proper circulation of the lymph, which allows the development of the typical small, clear, herpetiform pseudo vesicles.?
Although primary vulvar lymphangioma is reported to usually develop after birth and during early childhood, it can occur at any age, even in older patients. Congenital lesions usually develop as the patient grows and their external genitalia evolve, until lesions finally become clinically evident during adulthood or at any given age. ${ }^{6}$

Clinically, LC is characterized by the presence of small, thinwalled, translucent vesicles that range from 1 to $4 \mathrm{~mm}$ in size that converge forming vegetative lumps. These vesicles usually contain serous or crystalline liquid. Clinical presentation can also be asymptomatic. However, when symptoms present, they are usually described as vulvar itching, burning, pain, or foul discharge. Because of its clinical appearance and size, LC often results in aesthetic and psychosexual disorders.

Although it is widely acknowledged that LC is a benign pseudo tumor, treatment is recommended for symptomatic relief as well as improvement of aesthetic or psychological issues and discomfort. The psychosexual implications of this diagnosis are significant and cause great anxiety.

There is currently no established standard of care for LC. Treatments described in publications range from observation to vulvectomy. Lesion recurrence is frequent, regardless of treatment choice. Local excision is known to have a high rate of recurrence and treatment failure. Sclerotherapy and radiation have been reported as suitable options in the literature. Accompanying physical therapy is usually recommended in order to improve edema. Laser $\mathrm{CO}_{2}$ treatment has proven to be effective as long as it reaches the deep canaliculi. It also offers excellent cosmetic results when special care is taken in order to avoid development of keloids scarring.

Treatment selection usually depends on the location and size of the lesions. Observation is an option in asymptomatic patients who refuse treatment. Most reports opt for excisional surgery of the affected area. Regarding local excision, a $23.1 \%$ recurrence rate has been reported between 6 and 81 months after treatment. The recurrence rate seems to be higher when the initial lesion is greater than $7 \mathrm{~cm}$, despite radical excision. ${ }^{8,9}$ These findings correlate with our personal experience, as described in the first case of our series (Case 1).

\section{Conclusion}

Vulvar lymphangioma is a rare disease, which poses a great diagnostic challenge. It is important to consider this entity among our differential diagnoses in order to be able to offer adequate treatment. Although it is a benign condition, treatment is recommended in most cases, in order to address the cosmetic and psychosexual implications and avoid complications. There is no standard management for vulvar $\mathrm{LC}$, and despite treatment, recurrence is frequent. Most treatments have similar success and recurrence rates. Labiaplasty of the labia majora and laser $\mathrm{CO}_{2}$ vaporization are both adequate treatments, as well as conservative management (observation) in asymptomatic cases or according to patient preference.

\section{Acknowledgements}

The study was conducted in Buenos Aires, Argentina. This research did not receive any specific grant from funding agencies in the public, commercial, or not-for-profit sectors.

\section{Conflicts of interest}

The authors declare that they do not have any conflict of interest. 


\section{References}

1. Kim JK, Yoo KS, Moon JH, et al. Gallbladder lymphangioma: A case report and review of the literature. World $J$ grastroenterol. 2007;13(2):320-3.

2. Mehta V, Nayak S, Balachandran C, et al. Extensive congenital vulvar lymphangioma mimicking genital warts. Indian J dermatol. 2010;55(1):121-122.

3. Peachey RDG, Lim CC, Whimster IW. Lymphangioma of skin: A review of 65 cases. Br J Dermatol. 1970;83(5):519-27.

4. Akimoto K, Nogita T, Kawashima M. A case of acquired lymphangioma of the vulva. J Dermatol. 1993;20:449-51.
5. Amouri M, Masmoudi A, Boudaya S, et al. Acquired lymphangioma circumscriptum of the vulva.Dermatol Online J. 2007;13(4):10.

6. Vlastos AT, Malpica A, Follen M. Lymphangioma circumscriptum of the vulva: a review of the literature. Obstet Gynecol. 2003;101(5 Pt 1):946-54.

7. Uçmak D, Aytekin S, Sula B, et al. Acquired Vulvar Lymphangioma Circumscriptum. Case Rep Dermatol Med. 2013;2013:967890.

8. Kokcu A, Sari S, Kefeli M. Primary vulvar lymphangioma circumscriptum: a case reposrt and Review of literature. J Low Genit Tract Dis. 2015;19(1):e1-5.

9. Sasaki R, Negishi K, Akita H, et al. Successful treatment of congenital lymphangioma circumscriptum of the vulva with $\mathrm{CO} 2$ ang long-pulsed Nd:Yag lasers. Case Rep Dermatol. 2014;6:1-4. 